\section{IDDF2018-ABS-0130 THAI TRADITIONAL MASSAGE FOR DEFECATION STIMULATION IN BEDRIDDEN PATIENTS}

${ }^{1}$ Thubtai Saosiri", 'Ajjima Mongsin, 'Pilaipan Jantapasarn, ${ }^{2}$ Krit Pongpirul. 'Lue Amnat Hospital, Thailand; ${ }^{2}$ Chulalongkorn University, Thailand

\subsection{6/gutjnl-2018-IDDFabstracts.119}

Background Constipation is one of the most common uncomfortable conditions for bedridden patients. While conventional oral or rectal medications usually come with side effects, Thai traditional medicine presumed that constipation represents an imbalance of 'elements' and. This study aimed to share experience in using Thai traditional massage to stimulate defecation in bedridden patients.

Methods This retrospective study reviewed the medical records of the bedridden patients with constipation who underwent Royal Thai traditional massage, using standard 4 week in protocol. In the first 3 days of each week, a series of light massage (Basic Massage Line), 4-quadrant acupressure (Major Signal Points), and pressing over umbilicus are used in order to stimulate the bowel movement for $90 \mathrm{~min}$. Then a $30 \mathrm{~min}$ circular massage is done in the following 2 days. Defecation onset and frequency, patient compliance and comfort, as well as patient demographics were analysed and presented. Paired Student t-test was used to compare the number of interval days before and after the Thai traditional massage intervention. (Thai Clinical Trials Registry No. TCTR20170811005).

Results Twenty-three patients were included in this study; mean age 62 years, female $69.56 \%$, hypertension $91.30 \%$, diabetes mellitus $65.21 \%$, and both hypertension and diabetes mellitus 56.52\%. The distribution of Life Elements were Earth (Din) 34.78\%, Water (Nahm) 30.43\%, Wind (Lohm) 21.74\%, and Fire (Fire) $13.04 \%$. The patients had defecated at the interval of 8.217 days (SD 2.555) with the use of oral or rectal medication before participating in the study. The interval significantly reduced to 2.826 days (SD 0.995) after the Thai traditional massage protocol without a need for oral or rectal medications $(p<0.05)$. No side effects were observed.

Conclusions Thai traditional massage could safely help to stimulate defecation in bedridden patients.

\section{IDDF2018-ABS-0131 USEFULNESS OF CASTOR OIL FOR BOWEL PREPARATION FOR COLON CAPSULE ENDOSCOPY IN THE PATIENTS ON DIALYSIS}

Hotta Naoki*. Masuko Memorial Hospital Department of Capsule Endoscopy Center, Japan

\subsection{6/gutjpl-2018-IDDFabstracts. 120}

Background Colon capsule endoscopy was approved for reimbursement under the national health insurance system of Japan in 2014. For this reason, detailed examination by capsule endoscope appears as useful. However, the capsule excretion rate after recommended bowel preparation reportedly ranges $70 \%$ to $90 \%$, and administration of boosters is also necessary. For dialysis patients, liquid loading is a problem. In this study, we evaluated the efficacy of bowel preparation with castor oil for improving the capsule excretion rate. In 25 patients both capsule transit time and duration of colon capsule endoscopy were shorted. The results were presented and briefly discussed in the report.

Methods This study included patients who underwent colon capsule endoscopy at Masuko Memorial Hospital since March
2016. Colon Capsule endoscopy was performed with Pillcam Colon Capsule (Medtronic, Minneapolis, MN, USA). The recommended protocol for bowel preparation was modified (with the administration of castor oil), and success rate of completing entire colon observation was compared. The dose of castor oil was $6=$ at each time point. This study was approved by the ethics committee of my hospital, while we obtained written consent from the participants after providing a thorough explanation of the contents and methods of this study.

Results The success rate of completing entire colon observation was 24 patients were $35(96 \%)$ in the study. The maximal water intake of the fluid volume is $390=$. The average consumption in patients on dialysis was an average of median $317=$, and the patients of the smallest water intake had $200=$ two patients. A utility is very high for the patients on dialysis with moisture restrictions.

Conclusions Although the number of patients receiving bowel preparation with castor oil was small, entire colon observation was successful in dialysis patients. Thus, castor oil appears to be useful for bowel preparation for colon capsule endoscopy.

\section{IDDF2018-ABS-0134 VALIDATION OF THE ASIA PACIFIC COLORECTAL SCREENING (APCS) SCORE AND ITS MODIFIED VERSION IN PREDICTION OF ADVANCED COLORECTAL NEOPLASIA IN CHINESE ASYMPTOMATIC SUBJECTS}

${ }^{1}$ Martin Wong*, ${ }^{2}$ Ping Chen, ${ }^{2}$ Xiaoqin Yuan, ${ }^{2}$ Yunlin Wu, ${ }^{3}$ Shanjuan Wang, ${ }^{4}$ Wei Huang, ${ }^{5}$ Ming Hu, ${ }^{6}$ Tiansheng Huang, ${ }^{7}$ Xiaogang Li, ${ }^{8}$ Yinbing Tan. ${ }^{1}$ School of Public Health and Primary Care, CUHK, Hong Kong; ${ }^{2}$ Ruijin Hospital North, China; ${ }^{3}$ Hospital of Jiading District, Shanghai, China; ${ }^{4}$ Li Qun Hospital, Shanghai, China; ${ }^{5}$ Nan Xiang Hospital, Shanghai, China; ${ }^{6}$ Traditional Chinese Medicine Hospital of Jiading District, Shanghai, China; 'Dachang Hospital, Shanghai, China; ${ }^{8}$ Huaqiao Hospital, Kunshan, Jiangsu, China

\subsection{6/gutjnl-2018-IDDFabstracts.121}

Background The Asia-Pacific Colorectal Screening (APCS) score and a modified version that incorporates body mass index (BMI) has been widely recognised. This study aimed to compare the external validity of the APCS and the modified APCS to predict advanced colorectal neoplasia (ACN) in an asymptomatic Chinese population.

Methods A prospective, multi-centre colonoscopy study was conducted in 7 endoscopic units across North Shanghai, China from 2016 to 2017. Average-risk subjects were recruited during their attendance for screening colonoscopy. Information on the predictors, including age, gender, family history of colorectal cancer and smoking status were collected by self-reported questionnaires. Body weight and height were measured by trained professionals. All screening colonoscopy procedures were performed by senior colonoscopists. The histology of all resected lesions was reported using the same diagnostic criteria by pathologists who were blinded to the study design. Cumulative scores were derived for each subject and categorised as average, moderate and high risk. The risk of ACN was assessed for each risk tier and the excess risk in moderate and high-risk tiers was compared with subjects with average risk. The C-statistics was employed to evaluate the discriminatory capability of both APCS scores.

Results Among a total of 2813 enrolled subjects (mean age $58.7 \pm 8.4$ years), 679 cases $(24.1 \%)$ were found to have colorectal neoplasia, of which $142(5.0 \%)$ had ACN and 29 patients $(1.0 \%)$ had invasive cancers (table 1). In both APCS 